ISSN 1980-5098

\title{
INFLUÊNCIA DA PEDOFORMA NA COMPOSIÇÃO DO BANCO DE SEMENTES EM FLORESTA SECUNDÁRIA NA REGIÃO DE MAR DE MORROS, PINHEIRAL - RJ
}

\section{LANDFORM INFLUENCE IN THE SEED BANK COMPOSITION IN A SECONDARY FOREST IN 'MAR DE MORROS' REGION, PINHEIRAL-RJ STATE}

\begin{abstract}
Gilsonley Lopes dos Santos ${ }^{1}$ Marcos Gervasio Pereira ${ }^{2}$ Tiago de Conto ${ }^{3}$ Daniel Costa de Carvalho ${ }^{4}$ Thiago Azevedo de Amorim ${ }^{5}$ Victoria Maria Monteiro Mendonça ${ }^{6} \quad$ Luiz Fernando Duarte de Moraes ${ }^{7}$
\end{abstract}

\section{RESUMO}

A composição e qualidade do banco de sementes são alguns dos fatores responsáveis pela estrutura e composição florística dos ambientes florestais. O objetivo deste estudo foi avaliar o banco de sementes de um fragmento de Floresta Estacional Semidecidual, com influência das características topográficas da paisagem. Foram selecionadas duas pedoformas adjacentes com relevo do tipo convexa e côncava, sendo essas divididas em minissítios (MS) I, II, e III na pedoforma convexa, e IV, V, VI na pedoforma côncava. Foram amostrados 10 pontos de cada MS, distribuídos assistematicamente, com uma área superficial de $0,0625 \mathrm{~m}^{2}$ e profundidade de $3 \mathrm{~cm}$. Foi registrado um total de 4.862 sementes. $\mathrm{m}^{-1}$ germinadas no período de 90 dias, sendo na pedoforma convexa observado o maior número de sementes. $\mathrm{m}^{-1}$ germinadas. Em ambas as pedoformas, foram identificadas 35 famílias e 88 espécies; dentre as espécies, $37 \%$ têm hábito herbáceo; $24 \%$, arbóreo; $18 \%$, arbustivo; $12 \%$, trepador; e 2\%, epifítico. Quanto ao tipo de pedoforma, foram registradas $44 \%$ das espécies encontradas em ambas, $31 \%$ foram exclusivas da convexa e $25 \%$, da côncava. Para aos parâmetros florísticos e fitossociológicos, a pedoforma convexa apresentou maior número de sementes, famílias, espécies e riqueza, enquanto a côncava teve maiores índices de diversidade. As condições topográficas foram determinantes na composição florística e fitossociológica do banco de sementes, sendo os maiores valores de riqueza verificados na pedoforma convexa e de diversidade, na côncava, com maior índice de valor de importância para as famílias Asteraceae, Melastomataceae e Urticaceae.

Palavras-chave: Floresta Atlântica; relevo; fragmentos florestais.

\begin{abstract}
The composition and quality of the seed bank are some of the factors responsible for the structure and floristic composition of forest environments. The aim of this study was to evaluate the seed bank of a semideciduous forest patch, taking into account the influence of the landscape's topographical features. Two adjacent landforms, of convex and concave shapes, were selected. They were divided into mini sites

1 Engenheiro Florestal, MSc., Doutorando em Ciências Ambientais e Florestais, Universidade Federal Rural do Rio de Janeiro, BR 465, Km 7, CEP 23890-000, Seropédica (RJ), Brasil. leylopes85@hotmail.com

2 Engenheiro Agrônomo, Dr., Professor do Instituto de Agronomia, Universidade Federal Rural do Rio de Janeiro, BR 465, km 7, CEP 23890-000, Seropédica (RJ), Brasil. mgervasiopereira01@gmail.com

3 Engenheiro Florestal, Mestrando em Manejo Florestal, Swedish University of Agricultural Sciences (SLU), Sundsvägen 6, Alnarp, Suécia. tiagodc_89@yahoo.com.br

4 Engenheiro Florestal, Dr., em Ciências Ambientais e Florestais, Universidade Federal Rural do Rio de Janeiro, BR 465, Km 7, CEP 23890-000, Seropédica (RJ), Brasil. danielcostadecarvalho@gmail.com

5 Engenheiro Florestal, MSc., Doutorando em Ecologia e Evolução, Universidade do Estado do Rio de Janeiro, Rua São Francisco Xavier, 524, Maracanã, CEP 20550-013, Rio de Janeiro (RJ). azevedoamorim@hotmail.com

6 Acadêmica do Curso de Engenharia Florestal, Instituto de Florestas, Universidade Federal Rural do Rio de Janeiro, BR 465, KM 07, CEP 23890-000, Seropédica (RJ), Brasil. viic_monteiro@hotmail.com

7 Engenheiro Agrônomo, Dr., Pesquisador do Centro Nacional de Pesquisa de Agrobiologia, BR 465, km 7, CEP 23890-000, Seropédica (RJ), Brasil. luiz.moraes@embrapa.br
\end{abstract}

Recebido para publicação em 12/08/2014 e aceito em 12/07/2016 
(MS) I, II, and III in the convex landform and IV, V and VI in the concave one. Ten sample points on each MS were randomly distributed with a surface area of $0.0625 \mathrm{~m}^{2}$ and $3 \mathrm{~cm}$ deep. A total of 4,862 germinated seeds. $\mathrm{m}^{-1}$ was registered in 90 days, with the convex landform presenting the highest frequency of germinated seeds. $\mathrm{m}^{-1}$. A total of 35 families and 88 species were identified in both landforms, of which $37 \%$ were of herbaceous habit, $24 \%$ arboreal, $18 \%$ shrubs, $12 \%$ lianas and $2 \%$ epiphytic. Regarding the landform's shape, $44 \%$ of the species were found in both convex and concave ones, $31 \%$ were found exclusively on the convex landform and $25 \%$ exclusively on the concave one. Regarding floristic and phytosociological parameters, the convex landform accounted for a higher number of seeds, families, and species richness, while the concave landform accounted for higher diversity. Topography was a determinant factor for the floristic and phytosociological composition of the seed bank. The highest species richness was recorded in the convex landform and highest diversity in the concave landform, with Asteraceae, Melastomataceae and Urticaceae families presenting the highest importance indexes of value.

Keywords: Atlantic Forest; topography; forest fragment.

\section{INTRODUÇÃO}

A composição florística de um ambiente é resultante da interação das espécies com as condições ambientais e entre si, além da capacidade de dispersão dos indivíduos (HUBBELL, 2006). Esse processo de interação se inicia no banco de sementes, que é um dos responsáveis pela estrutura e composição florística dos ambientes em regeneração natural, por garantir a manutenção da diversidade das espécies, o estabelecimento dos grupos ecológicos e a restauração da sua riqueza (RODRIGUES; MARTINS; LEITE, 2010).

O banco de sementes do solo tem sua estrutura e a composição de espécies determinadas pelas características florísticas e pelos filtros ambientais, os quais determinam o recrutamento das espécies vegetais (FAIST; COLLINGE, 2015). No entanto, a composição e qualidade do banco de sementes são alguns dos fatores que garantem o potencial de resiliência dos ambientes que foram submetidos a processos de antropização (MARTINS et al., 2008).

O banco de sementes é composto por todas as sementes viáveis no solo ou associadas à serapilheira em uma determinada área em um dado momento (CALDATO et al., 1996), com influência das condições de temperatura, de precipitação e do efeito de borda (DIOGO et al., 2015). As sementes chegam ao ambiente por meio da chuva de sementes, dos agentes dispersores e pelos indivíduos vegetais que tiveram o papel de pioneiros na exploração do ambiente, os quais na maioria das vezes não estão mais presentes na composição florística atual (FRANCO et al., 2012).

As características do banco de sementes são influenciadas pelas condições topográficas, pois estudos como Havrdová, Douda e Doudová (2015) têm observado que a abundância e diversidade de espécies são influenciadas diretamente pela variação do relevo na paisagem. Nas Florestas Tropicais, a distribuição das espécies e a estrutura da vegetação e composição do banco de sementes têm mostrado uma relação positiva com as condições topográficas da paisagem, a qual é resultante da variação dos atributos químicos e físicos do solo (BENTOS; NASCIMENTO; WILLIAMSON, 2013). Os solos das regiões de encosta e topo apresentam menores teores de nutrientes, e os de baixadas, maiores teores de umidade e de nutrientes resultantes do acúmulo de matéria orgânica, sedimentos e água (SANTOS et al., 2010).

Diante disso, o objetivo deste estudo foi avaliar a composição e estrutura do banco de sementes de um fragmento de Floresta Estacional Semidecidual, com influência das características topográficas da paisagem na Região de Mar de Morros, Pinheiral - RJ.

\section{MATERIAL E MÉTODOS}

\section{Área de estudo}

O estudo foi realizado no município de Pinheiral, Rio de Janeiro, na região do Médio Paraíba Fluminense, na sub-bacia do Ribeirão Cachimbal, que compõe a bacia hidrográfica do rio Paraíba do Sul, localizado entre as latitudes $22^{\circ} 29^{\prime} 03^{\prime}$ 'S e $22^{\circ} 35^{\prime} 27^{\prime \prime}$ S e entre as longitudes $43^{\circ} 54^{\prime} 49^{\prime}$ ' W e $44^{\circ} 04^{\prime} 05^{\prime}$ 'W. 
Foram selecionadas duas pedoformas adjacentes com relevo do tipo convexa (convexa-divergente) e côncava (côncava-convergente) com orientação da vertente predominante a Sudeste e cobertura vegetal tipo Floresta Estacional Semidecidual Submontana (BRASIL, 1994; IBGE, 2012). As pedoformas foram segmentadas em três minissítios (MS) diferenciados quanto ao gradiente topográfico e à declividade, sendo: I (altitude média: $520 \mathrm{~m}$, declividade média: $45 \%$, largura: $20 \mathrm{~m}$, comprimento: $33 \mathrm{~m}$ ), II (altitude média: $530 \mathrm{~m}$, declividade média: $12 \%$, largura: $20 \mathrm{~m}$, comprimento: $30 \mathrm{~m}$ ) e III (altitude média: $550 \mathrm{~m}$, declividade média: $26 \%$, largura: $20 \mathrm{~m}$, comprimento: $72 \mathrm{~m}$ ) na pedoforma convexa, e IV (altitude média: $485 \mathrm{~m}$, declividade média: $30 \%$, largura: $20 \mathrm{~m}$, comprimento: $45 \mathrm{~m}$ ), V (altitude média: $590 \mathrm{~m}$, declividade média: $20 \%$, largura: $20 \mathrm{~m}$, comprimento: $30 \mathrm{~m}$ ) e VI (altitude média: $500 \mathrm{~m}$, declividade média: $32 \%$, largura: $20 \mathrm{~m}$, comprimento: $72 \mathrm{~m}$ ) na pedoforma côncava.

\section{Banco de sementes}

Para análise do banco de sementes do solo, foram amostrados 10 pontos em cada MS da pedoforma, distribuídos sistematicamente. Cada ponto apresentou uma área de $0,25 \mathrm{~m} \mathrm{x} 0,25 \mathrm{~m}\left(0,0625 \mathrm{~m}^{2}\right)$, na qual foram coletadas a serapilheira, juntamente com a camada superficial do solo até a profundidade de $3 \mathrm{~cm}$.

Posteriormente, as amostras foram homogeneizadas e divididas em dois tratamentos, sol (casa de vegetação) e sombra (casa de vegetação + sombrite 70\%). As amostras foram acondicionadas em bandejas $\left(0,033 \mathrm{~m}^{2}\right)$, totalizando 60 parcelas, que foram distribuídas aleatoriamente entre os tratamentos (sol e sombra), resultando em 30 parcelas para cada tratamento. Na bandeja, foi acondicionada uma camada de $1 \mathrm{~cm}$ de espessura de areia esterilizada, na qual posteriormente foram colocadas as amostras de solo e serapilheira contendo o banco de sementes. A irrigação foi realizada diariamente, sendo as bandejas furadas para drenagem da água em excesso.

A quantificação da germinação foi feita a cada 15 dias e até 90 dias após a instalação do experimento, sendo que as plântulas permaneceram nas bandejas ou sacos plásticos (repicagem) até um tamanho possível para sua identificação botânica. Para as espécies presentes no banco de sementes, foram calculados os parâmetros fitossociológicos: Densidade Absoluta (DA), Densidade Relativa (DR), Frequência Absoluta (FA), Frequência Relativa (FR), Índice de Valor de Importância do Banco de Sementes (IVIB), Índice de Shannon (H') e Índice de Equabilidade de Pielou (J'), conforme Magurran (2004).

\section{Identificação e classificação da vegetação}

As espécies vegetais presentes no banco de sementes do solo foram identificadas por meio de consultas à literatura botânica, a especialistas e comparadas com a coleção do herbário do Departamento de Botânica da Universidade Federal Rural do Rio de Janeiro (RBR). As espécies identificadas foram classificadas em grupos sucessionais (pioneiras, secundárias e clímax), adaptado segundo Gandolfi, Leitão Filho e Bezerra (1995), Lorenzi (2008; 2009a; 2009b) e Carvalho (2003; 2006; 2008; 2010).

\section{Análise dos dados}

Para comparar a germinação total das sementes e a germinação das sementes nos intervalos de tempo, foram realizadas análises de variâncias (ANOVA) fatoriais permutacionais (significância 5\%, 5000 permutações). A análise permutacional permitiu efetuar comparações sem que houvesse a necessidade de atender às premissas de normalidade e homogeneidade de variâncias. Os fatores das análises foram o tipo da pedoforma (convexa ou côncava) e o ambiente de germinação (sol ou sombra). De maneira similar, dentro de uma mesma pedoforma, foi realizada uma análise de variância fatorial, considerando os MS (três no total) e o ambiente de germinação.

Para todas as análises fatoriais, quando detectado algum valor significativo para um fator ou a interação entre fatores, foi realizado o teste de Tukey (significância 5\%), sendo verificada anteriormente a homogeneidade de variâncias com o teste de Levene. Em função da homogeneidade das variâncias, o teste de Tukey foi realizado sem que houvesse nenhuma transformação dos dados. 
Posteriormente, foi realizada a análise Adonis, com o objetivo de testar a existência de diferença na composição florística entre os tratamentos tipo da pedoforma e o ambiente de germinação (significância 5\%, 5000 permutações). Análise Adonis consiste em uma ANOVA que utiliza distância de matrizes como variável-resposta. Para a realização da análise, foi gerada uma matriz de abundância de espécies de cada unidade amostral. Em seguida, os valores brutos de abundância foram convertidos para abundância relativa. Foi calculada a dissimilaridade de Bray-Curtis entre cada unidade amostral. Essa matriz correspondeu a quão dissimilares as unidades amostrais eram quanto à composição florística. A comparação da composição florística apresentou dois fatores como variáveis preditoras: o tipo da pedoforma, o ambiente de germinação e a interação entre os fatores. Todas as análises foram realizadas no programa R 3.0.1 (R CORE TEAM, 2013), utilizando os pacotes ImPerm (WHEELER, 2010) e vegan (OKSANEN et al., 2013).

\section{RESULTADOS E DISCUSSÃO}

\section{Germinação}

Foi registrado um total de 4.862 sementes. $^{-1}$, germinadas ao final de 90 dias, sendo $74 \%$ registradas até o trigésimo dia após a instalação do experimento. Do total germinado, o maior número foi observado na pedoforma convexa $(\mathrm{gl}=1 ; \mathrm{F}=6,25 ; \mathrm{p}=0,01)$ (Figura 1); porém, em relação à disponibilidade de luz no ambiente de germinação, não foram verificadas diferenças estatísticas quanto ao número de sementes germinadas $(\mathrm{gl}=1 ; \mathrm{F}=1,25 ; \mathrm{p}=0,40)$ (Figura 1).

O número de sementes germinadas foi irregular e decrescente ao longo do tempo (Figura 2), sendo observadas diferenças estatísticas quanto ao tipo de pedoforma nos intervalos de 15 a 30 dias $(\mathrm{gl}=1 ; \mathrm{F}$ $=6,30 ; \mathrm{p}=0,006)$, e de 60 a 75 dias $(\mathrm{gl}=1 ; \mathrm{F}=4,46 ; \mathrm{p}=0,02)$. Os maiores valores germinados nesses intervalos ocorreram nas amostras da pedoforma convexa. O decréscimo do número de sementes germinadas em função do tempo possivelmente deve estar relacionado à diminuição da viabilidade das sementes do banco e à possível presença de semente em estágio de dormência (SANTOS, 2014).

Quando avaliado o número de sementes. $\mathrm{m}^{-2}$ germinadas entre os MS em uma mesma pedoforma, pode-se observar que na pedoforma convexa, o MS I diferiu estatisticamente do MS III ( $\mathrm{gl}=2 ; \mathrm{F}=3,67$; $\mathrm{p}=0,03$ ), com maior número de sementes germinadas no MS I (Figura 3). Em relação ao ambiente de germinação, houve diferença entre o MS I sombra para o MS II sol (gl=11, F=4,42; $\mathrm{p}=0,02)$, sendo maior o número de sementes germinadas em condições de sol. Já para a pedoforma côncava, não foram observadas diferenças significativas entre os MS, indicando ser este um ambiente mais homogêneo quanto às condições
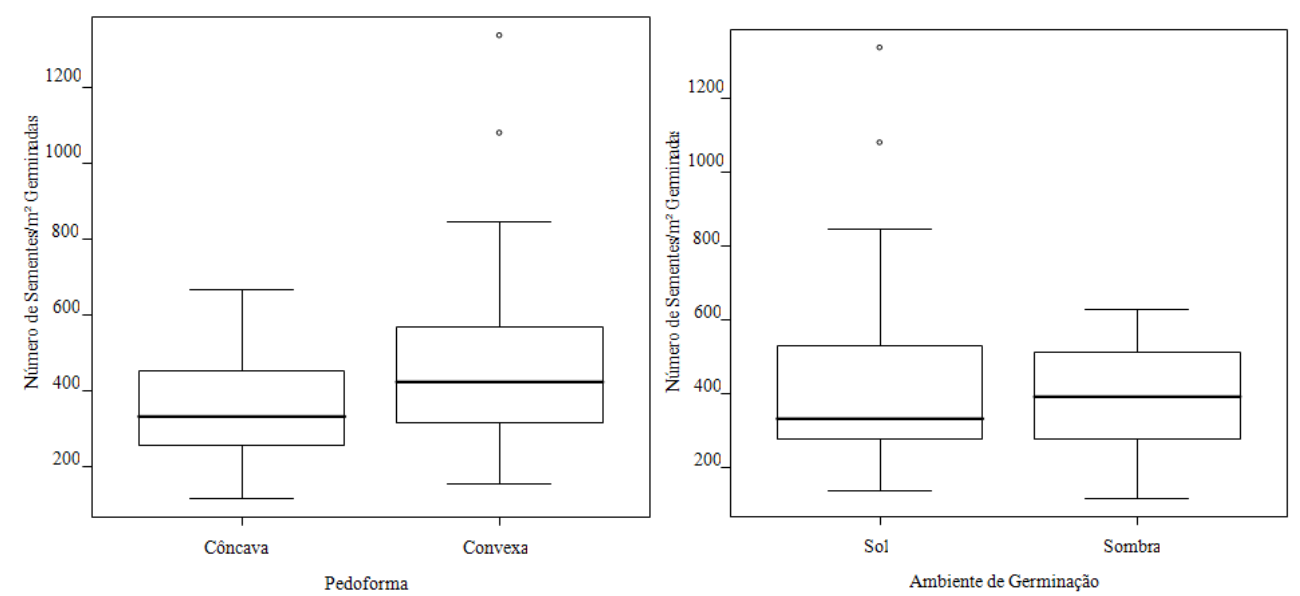

FIGURA 1: Número de sementes germinadas. $\mathrm{m}^{-1}$ do banco de sementes em relação ao tipo da pedoforma (côncava e convexa) e ao ambiente de germinação (sol e sombra) no período de 90 dias.

FIGURE 1: Number of germinated seeds $\mathrm{m}^{2}$ seed bank in relation to the type of landform (concave and convex) and to germination environment (sun and shade) in the period of 90 days. 


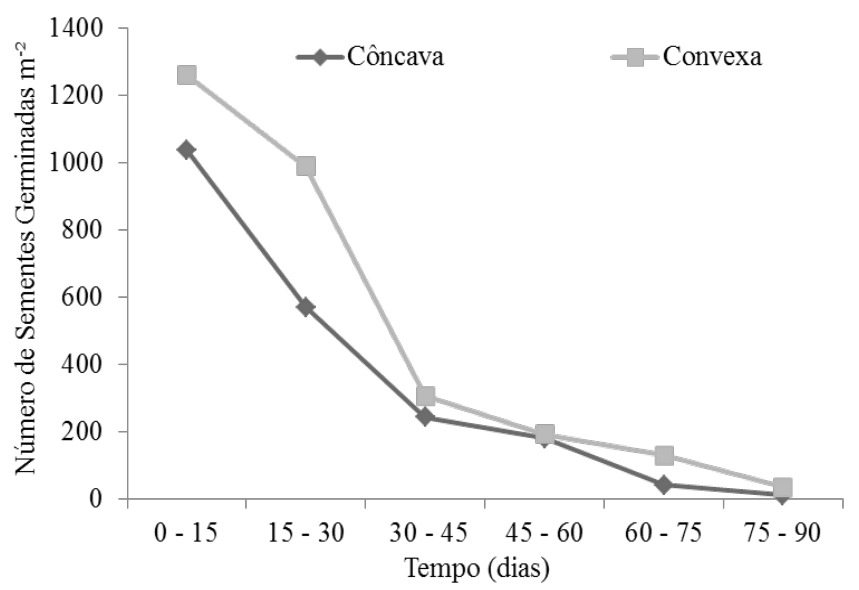

FIGURA 2: Número de sementes germinadas. $\mathrm{m}^{-2}$ do banco de sementes nas pedoformas convexa e côncava no período de 90 dias.

FIGURE 2: Number of germinated seeds $\mathrm{m}^{-2}$ of the seed bank in convex and concave landforms in the period of 90 days.

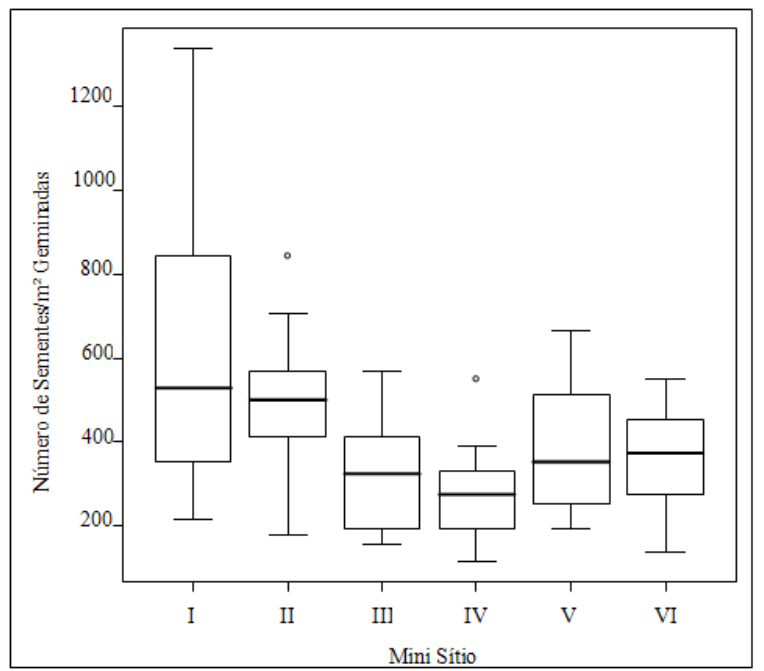

FIGURA 3: Número de sementes germinadas. $\mathrm{m}^{-2}$ por MS nas pedoformas convexa e côncava. Legenda: I, II e III - MS com variação do gradiente topográfico e da declividade na pedoforma convexa; IV, V e VI - MS com variação do gradiente topográfico e da declividade na pedoforma côncava, ao fim de 90 dias.

FIGURE 3: Number of germinated seeds $\mathrm{m}^{-2}$ for MS in convex and concave landforms. Legend: I, II and III - MS varying the topographic gradient and slope convex landform; IV, V and VI - MS with the topographic variation gradient and the gradient in the concave landform, after 90 days.

microclimáticas, bem como quanto à variação da declividade e gradiente topográfico.

O efeito das condições topográficas na densidade de sementes no solo também foi observado por Bentos, Nascimento e Williamson (2013), estudando banco de sementes do solo em uma Floresta Secundária Amazônica. Os autores observaram que, nos ambientes com relevo acidentado, o declive funciona como acelerador da perda de sementes do banco de sementes do solo (NASCIMENTO, 2009).

O maior número de sementes germinadas na pedoforma convexa pode ser explicado pelo estádio sucessional menos avançado em comparação à pedoforma côncava, pois, com o avanço do estádio sucessional, há um maior investimento na produção de plântulas e menor no banco de sementes. 
Padrão similar foi observado por Franco et al. (2012) quanto ao número de sementes germinadas e maior valor de germinação nos primeiros 30 dias, trabalhando com banco de sementes de um trecho de Floresta Estacional Semidecidual em Viçosa - MG. Por outro lado, o ambiente de germinação apresentou resultados diferentes dos observados nesse estudo, sendo o maior número de sementes germinadas observado pelos autores no ambiente sombreado, resultado que é discordante dos verificados neste estudo e por Figueiredo et al. (2011), que quantificaram maior germinação em ambiente a pleno sol.

O padrão de germinação quanto ao tipo de ambiente pode estar relacionado à adaptação das espécies a condições ambientais, o que pode ser observado nas Figuras 1, 2 e 3, em que o maior número de sementes germinadas por $\mathrm{m}^{-2}$ ocorreu nas amostras oriundas da pedoforma convexa, a qual na paisagem recebe uma maior incidência de luz comparada aos ambientes côncavos que se encontram em um estádio sucessional menos avançado. Estudos como os de Diogo et al. (2015), avaliando a deposição de sementes no gradiente borda para o interior de um fragmento de floresta tropical semidecídua no nordeste do Brasil, observaram que a variação das condições ambientais de temperatura e precipitação são determinantes no aporte de sementes.

\section{Composição florística e parâmetros fitossociológicos do banco de sementes}

Foram identificadas 35 famílias, 88 espécies e três sem identificação (Tabela 1), sendo $26 \%$ das espécies germinadas exclusivamente em condições de sombra e $24 \%$, em condições de sol; quanto às demais, $50 \%$ germinaram independentemente das condições do ambiente de germinação, o que se interpreta pelas condições ambientais que exercem influência na taxa de germinação das espécies do banco de sementes do solo.

As famílias de maior relevância no banco de sementes foram Asteraceae, Euphorbiaceae, Poaceae e Rubiaceae, padrão esse também observado nos estudos de Braga et al. (2008) e Franco et al. (2012).

Quanto ao hábito das espécies, observaram-se 37\% herbáceo, 24\% arbóreo, 18\% arbustivo, 12\% trepador e $2 \%$ epifítico, com $7 \%$ sem o hábito de vida identificado. Na classificação das espécies de acordo com o grupo sucessional, $48 \%$ foram consideradas pioneiras, $43 \%$ sem identificação e $9 \%$ secundárias (Tabela 1). Em se tratando de banco de sementes, é coerente que a maioria dos indivíduos presentes seja de herbáceos e pioneiros, o que para a regeneração natural garante o processo inicial de sucessão nos ambientes antropizados (GROMBONE-GUARATINI; RODRIGUES, 2002).

A distribuição das espécies quanto ao tipo de pedoforma obedeceu à seguinte ordem: $44 \%$ comum em ambas as pedoformas, com predominância das famílias Asteraceae e Poaceae; $31 \%$ exclusivas da pedoforma convexa, com predominância da família Asteraceae; e 25\% exclusivas da pedoforma côncava, com predominância da família Euphorbiaceae (Tabela 1).

O padrão de distribuição das espécies nas pedoformas pode estar relacionado à influência da variação do gradiente topográfico e da declividade na composição do banco de sementes e com as condições físicas e químicas do solo, como observado por Maia, Medeiros e Pillar (2004), Bentos, Nascimento e Williamson (2013) e Havrdová, Douda e Doudová (2015). Das espécies comuns em ambas as pedoformas, 58\% foram consideradas pioneiras e $43 \%$, sem identificação. O maior número de pioneiras é justificado pelo padrão observado na distribuição das espécies nos ambientes côncavo e convexo (Tabela 1).

Para as espécies exclusivas da pedoforma convexa, 11\% foram classificadas como secundárias; $50 \%$, pioneiras; e 39\%, não identificadas, sendo o processo de germinação das sementes influenciado tanto pelo ambiente de germinação quanto pelo MS (Tabela 1). Algumas espécies germinaram apenas em uma das condições dos ambientes de germinação, o que demostra o efeito do ambiente na germinação das sementes das espécies presentes no banco de sementes do solo.

Para as espécies exclusivas da pedoforma côncava, 9\% foram secundárias; $35 \%$, pioneiras; e $57 \%$, sem identificação, sendo o processo de germinação da espécie influenciado tanto pelo ambiente de germinação como pelo MS (Tabela 1). Os resultados observados são concordantes com o estádio sucessional avançado da vegetação, no qual as pioneiras tendem a estar mais presentes no banco de sementes do solo.

Quanto à diversidade das espécies, observou-se pela análise de distância beta que a composição florística entre as pedoformas difere estatisticamente $(\mathrm{gl}=2 ; \mathrm{F}=6,04 ; \mathrm{p}=0,0002)$, com maior número de espécies no ambiente convexo (Tabela 1). Já para as espécies de uma mesma pedoforma, não houve 
TABELA 1: Espécies vegetais presentes no banco de sementes do solo nas pedoformas convexa e côncava. TABLE 1: Plant species present in the soil seed bank in convex and concave landforms.

\begin{tabular}{|c|c|c|c|c|}
\hline Famílias e espécies & HB & GS & $\mathrm{P}$ & $\mathrm{AG}$ \\
\hline \multicolumn{5}{|l|}{ Amaranthaceae } \\
\hline Amaranthus hypochodriacus L. & $\mathrm{H}$ & $\mathrm{P}$ & $\mathrm{G}$ & $\mathrm{G}$ \\
\hline Gomphrena sp. & $\mathrm{H}$ & $\mathrm{P}$ & $\mathrm{CV}$ & $\mathrm{G}$ \\
\hline \multicolumn{5}{|l|}{ Annonaceae } \\
\hline Xylopia frutescens Aubl. & A & $\mathrm{S}$ & $\mathrm{CN}$ & SM \\
\hline \multicolumn{5}{|l|}{ Apocynaceae } \\
\hline Ditassa sp. & $\mathrm{T}$ & $\mathrm{P}$ & $\mathrm{G}$ & $\mathrm{G}$ \\
\hline Oxypetalum banksii R.Br. ex Schult. & $\mathrm{T}$ & $\mathrm{P}$ & $\mathrm{G}$ & $\mathrm{G}$ \\
\hline \multicolumn{5}{|l|}{ Araceae } \\
\hline Araceae sp1. & $\mathrm{E}$ & SI & G & $\mathrm{G}$ \\
\hline Araceae sp2. & $\mathrm{E}$ & SI & $\mathrm{G}$ & $\mathrm{G}$ \\
\hline \multicolumn{5}{|l|}{ Asteraceae } \\
\hline Asteraceae sp1. & $\mathrm{H}$ & SI & $\mathrm{G}$ & $\mathrm{S}$ \\
\hline Asteraceae sp2. & $\mathrm{H}$ & SI & $\mathrm{G}$ & $\mathrm{G}$ \\
\hline Asteraceae sp3. & $\mathrm{H}$ & SI & $\mathrm{G}$ & $\mathrm{G}$ \\
\hline Asteraceae sp4. & $\mathrm{H}$ & SI & $\mathrm{CN}$ & SM \\
\hline Austroeupatorium inulifolium (Kunth) King \& H.Rob. & Arb & $\mathrm{P}$ & $\mathrm{CV}$ & $\mathrm{S}$ \\
\hline Baccharis crispa Spreng. & Arb & $\mathrm{P}$ & $\mathrm{G}$ & $\mathrm{G}$ \\
\hline Baccharis serrulata (Lam.) Pers. & Arb & $\mathrm{P}$ & $\mathrm{CV}$ & SM \\
\hline Baccharis sp1. & $\mathrm{H}$ & SI & $\mathrm{CV}$ & SM \\
\hline Baccharis sp2. & $\mathrm{H}$ & SI & $\mathrm{G}$ & G \\
\hline Baccharis trinervis Pers. & $\mathrm{T}$ & $\mathrm{P}$ & $\mathrm{CV}$ & $\mathrm{S}$ \\
\hline Bidens pilosa $\mathrm{L}$. & $\mathrm{H}$ & $\mathrm{P}$ & $\mathrm{CV}$ & SM \\
\hline Chromolaena maximilianii (Schrad. ex DC.) R.M.King \& H.Rob. & Arb & $\mathrm{P}$ & $\mathrm{G}$ & $\mathrm{G}$ \\
\hline Conyza bonariensis (L.) Cronquist & $\mathrm{H}$ & $\mathrm{P}$ & $\mathrm{CV}$ & SM \\
\hline Cyrtocymura scorpioides (Lam.) H.Rob. & $\mathrm{T}$ & $\mathrm{P}$ & $\mathrm{CV}$ & SM \\
\hline Erechtites valerianifolius (Wolf) DC. & $\mathrm{H}$ & $\mathrm{P}$ & $\mathrm{G}$ & $\mathrm{G}$ \\
\hline Gamochaeta pensylvanica (Willd.) Cabrera & $\mathrm{H}$ & $\mathrm{P}$ & $\mathrm{G}$ & G \\
\hline Ipomoea sp. & SI & SI & $\mathrm{CN}$ & $\mathrm{S}$ \\
\hline Mikania hirsutissima DC. & $\mathrm{T}$ & $\mathrm{P}$ & $\mathrm{CV}$ & G \\
\hline Mikania micrantha Kunth & $\mathrm{T}$ & $\mathrm{P}$ & $\mathrm{G}$ & $\mathrm{S}$ \\
\hline Piptocarpha leprosa (Less.) Baker & $\mathrm{T}$ & $\mathrm{P}$ & $\mathrm{G}$ & $\mathrm{G}$ \\
\hline Vernonia cinerea (L.) Less. & $\mathrm{H}$ & $\mathrm{P}$ & $\mathrm{G}$ & $\mathrm{S}$ \\
\hline \multicolumn{5}{|l|}{ Bignoniaceae } \\
\hline Handroanthus sp. & A & SI & $\mathrm{CV}$ & $\mathrm{G}$ \\
\hline Jacaranda montana Morawetz & A & $\mathrm{S}$ & $\mathrm{CV}$ & $\mathrm{G}$ \\
\hline \multicolumn{5}{|l|}{ Cannabaceae } \\
\hline Trema micrantha (L.) Blume & A & $\mathrm{P}$ & $\mathrm{G}$ & $\mathrm{G}$ \\
\hline \multicolumn{5}{|l|}{ Caryophyllaceae } \\
\hline Caryophyllaceae sp. & $\mathrm{H}$ & SI & $\mathrm{CN}$ & $\mathrm{G}$ \\
\hline \multicolumn{5}{|l|}{ Commelinaceae } \\
\hline Commelina diffusa Burm.f. & $\mathrm{H}$ & $\mathrm{P}$ & $\mathrm{CN}$ & $\mathrm{S}$ \\
\hline Dichorisandra thyrsiflora J.C.Mikan & $\mathrm{H}$ & $\mathrm{P}$ & $\mathrm{CN}$ & SM \\
\hline \multicolumn{5}{|l|}{ Convolvulaceae } \\
\hline Ipomoea sp. & $\mathrm{T}$ & SI & $\mathrm{G}$ & $\mathrm{G}$ \\
\hline \multicolumn{5}{|l|}{ Euphorbiaceae } \\
\hline Actinostemon sp. & A & SI & $\mathrm{CN}$ & SM \\
\hline Alchornea triplinervia (Spreng.) Müll.Arg. & A & $\mathrm{P}$ & G & G \\
\hline Croton sp1. & Arb & SI & $\mathrm{CN}$ & SM \\
\hline Croton $\mathrm{sp} 2$. & Arb & SI & $\mathrm{CN}$ & $\mathrm{S}$ \\
\hline Euphorbia hyssopifolia L. & $\mathrm{H}$ & $\mathrm{P}$ & $\mathrm{CV}$ & $\mathrm{G}$ \\
\hline Maprounea guianensis Aubl. & A & $\mathrm{S}$ & $\mathrm{CV}$ & SM \\
\hline \multicolumn{5}{|l|}{ Fabaceae } \\
\hline Schizolobium parahyba (Vell.) Blake & A & $\mathrm{P}$ & $\mathrm{CV}$ & $\mathrm{S}$ \\
\hline \multicolumn{5}{|l|}{ Hypoxidaceae } \\
\hline Hypoxis decumbens $\mathrm{L}$. & $\mathrm{H}$ & $\mathrm{P}$ & $\mathrm{G}$ & $\mathrm{G}$ \\
\hline \multicolumn{5}{|l|}{ Indeterminada } \\
\hline Indeterminada 1 & SI & SI & $\mathrm{CV}$ & SM \\
\hline
\end{tabular}


TABELA 1: Continuação...

TABLE 1: Continued...

\begin{tabular}{|c|c|c|c|c|}
\hline Famílias e espécies & $\mathrm{HB}$ & GS & $\mathrm{P}$ & $\mathrm{AG}$ \\
\hline Indeterminada 2 & SI & SI & $\mathrm{G}$ & SM \\
\hline Indeterminada 3 & SI & SI & $\mathrm{CN}$ & $\mathrm{S}$ \\
\hline \multicolumn{5}{|l|}{ Lamiaceae } \\
\hline Lamiaceae sp. & $\mathrm{H}$ & SI & $\mathrm{G}$ & $\mathrm{S}$ \\
\hline \multicolumn{5}{|l|}{ Lauraceae sp. } \\
\hline Lauraceae sp. & A & SI & $\mathrm{CV}$ & SM \\
\hline \multicolumn{5}{|l|}{ Malpighiaceae } \\
\hline Byrsonima sericea DC. & $\mathrm{A}$ & $\mathrm{P}$ & $\mathrm{CV}$ & $\mathrm{S}$ \\
\hline \multicolumn{5}{|l|}{ Malvaceae } \\
\hline Luehea sp. & A & SI & $\mathrm{CV}$ & $\mathrm{S}$ \\
\hline Sida acuta Burm.f. & $\mathrm{H}$ & $\mathrm{P}$ & $\mathrm{CN}$ & $\mathrm{S}$ \\
\hline Sida rhombifolia L. & $\mathrm{H}$ & $\mathrm{P}$ & $\mathrm{CV}$ & $\mathrm{S}$ \\
\hline \multicolumn{5}{|l|}{ Marantaceae } \\
\hline Ctenanthe sp. & $\mathrm{H}$ & SI & $\mathrm{CN}$ & SM \\
\hline \multicolumn{5}{|l|}{ Melastomataceae } \\
\hline Clidemia hirta (L.) D.Don & Arb & $\mathrm{P}$ & $\mathrm{G}$ & $\mathrm{G}$ \\
\hline Miconia calvescens DC. & A & $\mathrm{P}$ & G & $\mathrm{G}$ \\
\hline Tibouchina sp. & Arb & SI & $\mathrm{G}$ & $\mathrm{G}$ \\
\hline \multicolumn{5}{|l|}{ Moraceae } \\
\hline Ficus arpazusa Casar. & $\mathrm{A}$ & $\mathrm{S}$ & $\mathrm{G}$ & $\mathrm{G}$ \\
\hline Ficus eximia Schott & A & $\mathrm{S}$ & $\mathrm{CV}$ & SM \\
\hline Ficus sp. & A & SI & $\mathrm{CN}$ & $\mathrm{G}$ \\
\hline \multicolumn{5}{|l|}{ Myrtaceae } \\
\hline Myrciaria sp. & $\mathrm{A}$ & SI & $\mathrm{CV}$ & SM \\
\hline \multicolumn{5}{|l|}{ Oxalidaceae } \\
\hline Oxalis sp. & $\mathrm{H}$ & SI & $\mathrm{CV}$ & $\mathrm{G}$ \\
\hline \multicolumn{5}{|l|}{ Phyllanthaceae } \\
\hline Phyllanthus tenellus Roxb. & $\mathrm{H}$ & $\mathrm{P}$ & $\mathrm{G}$ & $\mathrm{G}$ \\
\hline \multicolumn{5}{|l|}{$\begin{array}{l}\text { Piperaceae } \\
\end{array}$} \\
\hline Piper dilatatum Rich. & Arb & $\mathrm{S}$ & G & $\mathrm{G}$ \\
\hline Piper sp. & Arb & SI & $\mathrm{G}$ & $\mathrm{G}$ \\
\hline \multicolumn{5}{|l|}{ Plantaginaceae } \\
\hline Plantaginaceae sp. & $\mathrm{H}$ & SI & $\mathrm{CV}$ & SM \\
\hline \multicolumn{5}{|l|}{ Poaceae } \\
\hline Eleusine indica (L.) Gaertn. & $\mathrm{H}$ & $\mathrm{P}$ & $\mathrm{G}$ & $\mathrm{G}$ \\
\hline Olyra latifolia $\mathrm{L}$. & $\mathrm{H}$ & $\mathrm{P}$ & G & $\mathrm{G}$ \\
\hline Pharus lappulaceus Aubl. & $\mathrm{H}$ & $\mathrm{P}$ & $\mathrm{G}$ & $\mathrm{G}$ \\
\hline Poaceae sp1. & $\mathrm{H}$ & SI & $\mathrm{CV}$ & $\mathrm{S}$ \\
\hline Poaceae sp2. & $\mathrm{H}$ & SI & $\mathrm{G}$ & $\mathrm{S}$ \\
\hline Urochloa brizantha (Hochst. ex A. Rich.) R.D.Webster & $\mathrm{H}$ & $\mathrm{P}$ & $\mathrm{G}$ & SM \\
\hline \multicolumn{5}{|l|}{ Polygonaceae } \\
\hline Coccoloba sp. & $\mathrm{T}$ & SI & $\mathrm{G}$ & $\mathrm{G}$ \\
\hline \multicolumn{5}{|l|}{ Primulaceae } \\
\hline Myrsine coriacea (Sw.) R.Br. ex Roem. \& Schult. & A & $\mathrm{P}$ & $\mathrm{G}$ & $\mathrm{S}$ \\
\hline \multicolumn{5}{|l|}{ Rhamnaceae } \\
\hline Gouania ulmifolia Hook. \& Arn. & $\mathrm{T}$ & $\mathrm{P}$ & $\mathrm{CV}$ & $\mathrm{G}$ \\
\hline \multicolumn{5}{|l|}{ Rosaceae } \\
\hline Rubus brasiliensis Mart. & Arb & $\mathrm{P}$ & $\mathrm{G}$ & $\mathrm{G}$ \\
\hline Rubiaceae & & & & \\
\hline Faramea sp. & Arb & SI & $\mathrm{G}$ & $\mathrm{G}$ \\
\hline Galium sp. & $\mathrm{H}$ & SI & $\mathrm{CN}$ & $\mathrm{S}$ \\
\hline Psychotria carthagenensis Jacq. & A & $\mathrm{S}$ & $\mathrm{CV}$ & SM \\
\hline Psychotria sp1. & Arb & SI & $\mathrm{CN}$ & $\mathrm{S}$ \\
\hline Psychotria sp2. & Arb & SI & $\mathrm{G}$ & $\mathrm{G}$ \\
\hline Rubiaceae sp1. & & SI & $\mathrm{CV}$ & $\mathrm{G}$ \\
\hline Rubiaceae sp2. & & SI & $\mathrm{CN}$ & $\mathrm{G}$ \\
\hline Rudgea sp. & A & SI & $\mathrm{CV}$ & $\mathrm{S}$ \\
\hline Sabicea sp. & $\mathrm{T}$ & SI & $\mathrm{G}$ & $\mathrm{G}$ \\
\hline
\end{tabular}

Continua... 
TABELA 1: Continuação...

TABLE 1: Continued...

\begin{tabular}{|c|c|c|c|c|}
\hline Famílias e espécies & $\mathrm{HB}$ & GS & $\mathrm{P}$ & $\mathrm{AG}$ \\
\hline \multicolumn{5}{|l|}{ Rutaceae } \\
\hline Zanthoxylum rhoifolium Lam. & A & $\mathrm{S}$ & $\mathrm{CN}$ & SM \\
\hline Scrophulariaceae & & & & \\
\hline Buddleja stachyoides Cham. \& Schltdl. & $\mathrm{H}$ & $\mathrm{P}$ & $\mathrm{CN}$ & $\mathrm{G}$ \\
\hline \multicolumn{5}{|l|}{ Solanaceae } \\
\hline Solanum asperum Rich. & Arb & $\mathrm{P}$ & $\mathrm{CN}$ & $\mathrm{G}$ \\
\hline Solanum paniculatum $\mathrm{L}$. & Arb & $\mathrm{P}$ & $\mathrm{G}$ & $\mathrm{G}$ \\
\hline Solanum swartzianum Roem. \& Schult. & Arb & $\mathrm{P}$ & $\mathrm{CN}$ & $\mathrm{G}$ \\
\hline \multicolumn{5}{|l|}{ Urticaceae } \\
\hline Cecropia pachystachya Trecul & Arb & $\mathrm{P}$ & G & $\mathrm{G}$ \\
\hline Urera nitida (Vell.) P.Brack & Arb & $\mathrm{P}$ & $\mathrm{CN}$ & $\mathrm{S}$ \\
\hline
\end{tabular}

Em que: HB: Hábito (A: Arbóreo, Arb: Arbustivo, H: Herbáceo, T: Trepador e SI: Sem identificação), GS: Grupo sucessional (P: Pioneira, S: Secundária e SI: Sem identificação), P: Pedoforma (CV: Convexa, CN: Côncava e G: Ocorreu nas duas pedoformas) e AG: Ambiente de Germinação (S: Sol, SM: Sombra e G: Ocorreu nos dois ambientes).

diferença entre os MS em ambos os ambientes, convexo e côncavo.

$\mathrm{Na}$ distribuição das espécies quanto ao valor de importância no banco de sementes (IVIB), as famílias Asteraceae, Melastomataceae e Urticaceae se destacaram com as espécies Erechtites valerianifolius, Clidemia hirta, Miconia calvescens, Tibouchina sp. e Cecropia pachystachya (Tabela 2), sendo essas de ocorrência em ambas as pedoformas e MS. Quanto aos MS, as espécies que se destacaram foram no I, Clidemia hirta, Hypoxis decumbens e Cecropia pachystachya; no II, Cecropia pachystachya, Miconia calvescens e Clidemia hirta; no III, Cecropia pachystachya, Miconia calvescens e Clidemia hirta; no , Clidemia hirta, Cecropia pachystachya e Tibouchina sp.; no V, Clidemia hirta, Cecropia pachystachya e Tibouchina sp.; e no VI, Cecropia pachystachya, Clidemia hirta e Tibouchina sp..

Diversos são os estudos em que as famílias Asteraceae, Melastomataceae e Urticaceae são as que têm maior número de espécies no banco de sementes (BRAGA et al., 2008; FIGUEIREDO et al., 2011; FRANCO et al., 2012, CAMARGOS et al., 2013), sendo que neste estudo estas foram as famílias que apresentaram maiores valores de índice de importância no banco de sementes em todos os MS avaliados.

Foram constatadas diferenças entre os parâmetros florísticos e fitossociológicos quanto ao ambiente de germinação. No ambiente de germinação sol, foi observada uma maior riqueza de famílias e espécies, enquanto no ambiente de sombra, maior número de indivíduos, maiores índices de Shannon e de equabilidade (Tabela 3). Portanto, verificou-se que a germinação em ambiente de sombra proporcionou uma maior diversidade e melhor distribuição das espécies germinadas.

Quanto aos parâmetros florísticos e fitossociológicos, a pedoforma convexa apresentou maior riqueza de famílias e de espécies em relação à côncava. Enquanto na pedoforma côncava, foram observados maiores índices de diversidade e de equabilidade das espécies germinadas (Tabela 3). Essas diferenças entre riqueza, diversidade e equabilidade estão relacionadas às características ambientais proporcionadas pelas diferentes pedoformas.

Para os parâmetros florísticos e fitossociológicos nos MS, pode-se observar que, com o aumento do gradiente topográfico e a variação da declividade dentro da pedoforma, alteram-se a estrutura, a riqueza e a diversidade das espécies presentes no banco de sementes. Nesse sentido, observa-se a maior riqueza nos MS situados na parte inferior das pedoformas; contudo, foi maior a diversidade ( $\left.\mathrm{H}^{\prime}\right)$ e melhor distribuição das espécies (J') nos MS situados na parte superior das pedoformas (Tabela 3).

Portanto, na análise da Tabela 3, observa-se a importância de se considerar os microambientes no planejamento ambiental. Esse planejamento ganha ainda mais importância quando estiver relacionado aos processos de recuperação de ambientes antropizados.

Buscando entender a variação do banco de sementes em função dos fatores ambientais que influenciam no desenvolvimento e distribuição da vegetação, Maia, Medeiros e Pillar (2004) também observaram o efeito do relevo na composição e tamanho do banco de sementes. Os autores determinaram 
TABELA2: Distribuição das 20 espécies com maior IVIB quanto aos parâmetros florísticos e fitossociológicos do banco de sementes nas pedoformas convexa e côncava.

TABLE 2: Distribution of 20 species with larger IVIB regarding the floristic and phytosociological parameters of the seed bank in convex and concave landforms.

\begin{tabular}{|c|c|c|c|c|}
\hline Espécies & $\mathrm{N}$ & DR & FR & IVIB \\
\hline Clidemia hirta & 235 & 20,09 & 9,11 & 29,19 \\
\hline Cecropia pachystachya & 239 & 20,43 & 8,41 & 28,83 \\
\hline Miconia calvescens & 101 & 8,63 & 6,3 & 14,94 \\
\hline Erechtites valerianifolius & 53 & 4,53 & 5,43 & 9,96 \\
\hline Tibouchina sp. & 53 & 4,53 & 3,85 & 8,38 \\
\hline Hypoxis decumbens & 47 & 4,02 & 2,8 & 6,82 \\
\hline Chromolaena maximilianii & 28 & 2,39 & 2,98 & 5,37 \\
\hline Araceae sp1. & 52 & 4,44 & 0,53 & 4,97 \\
\hline Trema micrantha & 22 & 1,88 & 2,63 & 4,51 \\
\hline Sabicea sp. & 22 & 1,88 & 2,28 & 4,16 \\
\hline Araceae sp2. & 16 & 1,37 & 1,93 & 3,29 \\
\hline Caryophyllaceae sp. & 22 & 1,88 & 1,4 & 3,28 \\
\hline Eleusine indica & 13 & 1,11 & 2,1 & 3,21 \\
\hline Amaranthus hypochodriacus & 15 & 1,28 & 1,58 & 2,86 \\
\hline Buddleja stachyoides & 11 & 0,94 & 1,75 & 2,69 \\
\hline Psychotria sp2. & 9 & 0,77 & 1,75 & 2,52 \\
\hline Asteraceae sp1. & 8 & 0,68 & 1,4 & 2,08 \\
\hline Ditassa sp. & 10 & 0,85 & 1,23 & 2,08 \\
\hline Handroanthus sp. & 7 & 0,6 & 1,4 & 2 \\
\hline Ipomoea sp. & 7 & 0,6 & 1,4 & 2 \\
\hline Piper sp. & 7 & 0,6 & 1,4 & 2 \\
\hline
\end{tabular}

Em que: N: Número de indivíduos, DR: Densidade relativa, FR: Frequência relativa, IVIB: Índice de valor de Importância no banco de sementes.

TABELA 3: Análises florística e fitossociológica do banco de semente das pedoformas convexa e côncava. TABLE 3: Floristic and phytosociological analysis of the seed bank in convex and concave landforms.

\begin{tabular}{cccccc}
\hline Área de Estudo & $\mathrm{N}$ & $\mathrm{N}^{\mathrm{o}}$ de Famílias & $\mathrm{N}^{\mathrm{o}}$ de Espécies & $\mathrm{H}$ & $\mathrm{J}$ \\
\hline \multicolumn{7}{c}{ ' } \\
\hline Convexa & 720 & 30 & 69 & 2,74 & 0,65 \\
Côncava & 450 & 29 & 63 & 3,12 & 0,75 \\
\hline \multicolumn{7}{c}{ Ambiente de Germinação } \\
\hline Sol & 523 & 32 & 69 & 2,89 & 0,68 \\
Sombra & 647 & 30 & 63 & 3,07 & 0,72 \\
\hline I & 319 & 22 & Minissítios & 0,66 \\
II & 256 & 20 & 40 & 2,44 & 0,63 \\
III & 145 & 13 & 43 & 2,39 & 0,69 \\
IV & 148 & 17 & 33 & 2,26 & 0,78 \\
V & 142 & 20 & 35 & 2,72 & 0,83 \\
VI & 160 & 18 & 37 & 2,93 & 0,87 \\
\hline
\end{tabular}

Em que: N (número de indivíduos); H' (índice de diversidade de Shannon); J' (índice de equabilidade); I, II e III - MS com variação do gradiente topográfico e da declividade na pedoforma côncava; IV, V e VI - MS a variação do gradiente topográfico e da declividade na pedoforma convexa. 
que os fatores ambientais de maior relevância foram a posição do relevo e as variáveis correlacionadas como: o grau de umidade, teores de nutrientes, argila e matéria orgânica do solo.

\section{CONCLUSÕES}

As condições topográficas são determinantes na composição do banco de sementes, sendo o ambiente convexo o que apresenta maior número de sementes no solo em comparação ao côncavo;

A composição florística do banco de sementes é influenciada pelo tipo de pedoforma, sendo os maiores valores de riqueza verificados na pedoforma convexa, e os maiores valores de diversidade, na pedoforma côncava;

As famílias Asteraceae, Melastomataceae e Urticaceae foram as que apresentaram as espécies de maior índice de valor de importância no banco de sementes do solo, sendo essas espécies de relevante importância nos processos de regeneração na região de Mar de Morros.

\section{AGRADECIMENTOS}

Ao PPGCAF, À FAPERJ, Ao IFRJ - Campus Pinheiral, Ao Instituto de Florestas da UFRRJ, À CAPES e Ao CNPq.

\section{REFERÊNCIAS}

BENTOS, T. V.; NASCIMENTO, H. E. M.; WILLIAMSON, G. B. Tree seedling recruitment in Amazon secondary forest: Importance of topography and gap micro-site conditions. Forest Ecology and Management, Amsterdam, v. 287, p. 140-146, 2013.

BRAGA, A. J. T. et al. Composição do banco de sementes de uma Floresta Semidecidual Secundária considerando o seu potencial de uso para recuperação ambiental. Revista Árvore, Viçosa, MG, v. 32, n. 6, p. 1089-1098, 2008.

BRASIL. Ministério do Meio Ambiente. Resolução 006 de 4 de maio de 1994. Disponível em: <http:// www.mma.gov.br/conama/legiano1.cfm>. Acesso em: 20 maio 2012.

CALDATO, S. L. et al. Estudo da regeneração natural, banco de sementes e chuva de sementes na reserva genética florestal de Caçador, SC. Ciência Florestal, Santa Maria, v. 6, n. 1, p. 27-38, 1996.

CAMARGOS, V. L. et al. Influência do fogo no banco de sementes do solo em Floresta Estacional Semidecidual. Ciência Florestal, Santa Maria, v. 23, n. 1, p. 19-28, jan./mar. 2013.

CARVALHO, P. E. R. Espécies Arbóreas Brasileiras. Brasília: Embrapa Informação Tecnológica; Colombo: Embrapa Florestas, 2003. v. 1, 1039 p.

CARVAlHO, P. E. R. Espécies Arbóreas Brasileiras. Brasília: Embrapa Informação Tecnológica; Colombo: Embrapa Florestas, 2006. v. 2, 627 p.

CARVALHO, P. E. R. Espécies Arbóreas Brasileiras. Brasília: Embrapa Informação Tecnológica; Colombo: Embrapa Florestas, 2008. v. 3, 593 p.

CARVALHO, P. E. R. Espécies Arbóreas Brasileiras. Brasília: Embrapa Informação Tecnológica; Colombo: Embrapa Florestas, 2010. v. 4, 644 p.

DIOGO, I. J. S. et al. Seed deposition in the edge-interior gradient of a degraded fragment of tropical semideciduos forest, Northeastern Brazil. Revista de Biología Tropical, San José, v. 63, n. 4, p. 981-994, 2015.

FAIST, A. M.; COLLINGE, S. K. Seed bank composition varies along invasion and inundation gradients in vernal pool wetlands. Plant Ecology, Dordrecht, v. 216, n. 4, p. 553-564, 2015.

FIGUEIREDO, P. H. A. et al. Avaliação do potencial seminal da Cecropia pachystachya Trécul no banco de sementes do solo de um fragmento florestal em restauração espontânea na Mata Atlântica Pinheiral-RJ.

Revista de Biociências da Universidade de Taubaté, São Paulo, v. 17, n. 2, p. 43-51, 2011.

FRANCO, B. K. S. et al. Densidade e composição florística do banco de sementes de um trecho de Floresta Estacional Semidecidual no campus da Universidade Federal de Viçosa. Revista Árvore, Viçosa, MG, v. 36, n. 3, p. 423-432, 2012. 
GANDOLFI, S.; LEITÃO FILHO, H. F.; BEZERRA, C. L. E. Levantamento florístico e caráter sucessional das espécies arbustivo arbóreas de uma floresta mesófila semidecídua no município de Guarulhos, SP. Revista Brasileira de Biologia, São Carlos, v. 55, n. 4, p. 753-767, 1995.

GROMBONE-GUARATINI, M. T.; RODRIGUES, R. R. Seed bank and seed rain in a seasonal SemiDeciduous Forest in South-Eastern Brazil. Journal of Tropical Ecology, Winchelsea, v. 18, p. 759-774, 2002.

HAVRDOVÁ, A.; DOUDA, J.; DOUDOVÁ, J. Local topography affects seed bank successional patterns in alluvial meadows. Flora, Jena, v. 217, p. 155-163, 2015.

HUBBELL, S. P. Neutral theory and the evolution of ecological equivalence. Ecology, New York, v. 87, n. 6, p. 1387-1398, 2006.

IBGE. Manual técnico da vegetação brasileira: sistema fitogeográfico Inventário das formações florestais e campestres, técnicas e manejo de coleções botânicas, procedimentos para mapeamentos. 2. ed. Rio de Janeiro: IBGE, 2012. $271 \mathrm{p}$.

LORENZI, H. Árvores Brasileiras. Manual de identificação e cultivo de plantas arbóreas nativas do Brasil. 5. ed. Nova Odessa: Instituto Plantarum, 2008. v. 1, 384 p.

LORENZI, H. Árvores Brasileiras. Manual de identificação e cultivo de plantas arbóreas nativas do Brasil. 3. ed. Nova Odessa: Instituto Plantarum, 2009a. v. 2, 384 p.

LORENZI, H. Árvores Brasileiras. Manual de identificação e cultivo de plantas arbóreas nativas do Brasil. 1. ed. Nova Odessa: Instituto Plantarum, 2009b. v. 3, 384 p.

MAIA, F. C.; MEDEIROS, R. B. PILLAR, V. P. Focht, telmo. soil seed bank variation patterns according to environmental factors in a natural grassland. Revista Brasileira de Sementes, Londrina, v. 26, n. 2, p. 126-137, 2004.

MAGURRAN, A. E. Measuring biological diversity. Oxford: Blackwell Science, 1994. 256 p.

MARTINS, S. V. et al. Banco de sementes como indicador de restauração de uma área degradada por mineração de caulim em Brás Pires, MG. Revista Árvore, Viçosa, MG, v. 32, n. 6, p. 1081-1088, 2008.

NASCIMENTO, J. G. C. Influência da erosão na regeneração de clareiras. 2009. 135 f. Tese (Doutorado) - Instituto Nacional de Pesquisa da Amazônia, Manaus, 2009.

OKSANEN, J. et al. Vegan: Community Ecology Package. R package version 2.0-10. 2013. Disponível em: $<$ http://CRAN.R-project.org/package= $=$ vegan $>$.

R CORE TEAM. R: A language and environment for statistical computing. Vienna: R Foundation for Statistical Compuing, 2013. Disponível em: <http://www.R-project.org/>.

RODRIGUES, B. D.; MARTINS, S. V.; LEITE, H. G. Avaliação do potencial da transposição da serapilheira e do banco de sementes do solo para restauração florestal em áreas degradas. Revista Árvore, Viçosa, MG, v. 34, n. 1 , p. $65-73,2010$.

SANTOS, A. C. et al. Gênese e classificação de solos numa topossequência no ambiente de Mar de Morros do médio vale Paraíba do Sul, RJ. Revista Brasileira de Ciência do Solo, Viçosa, MG, v. 34, p. 1297-1314, 2010.

SANTOS, G. L. Efeito da Pedoforma no Processo de Sucessão Secundária em Fragmentos Florestais na Região do Médio Vale do Paraíba do Sul, Pinheiral, RJ. 2014. 130 f. Dissertação (Mestrado em Ciências Ambientais e Florestais) - Universidade Federal Rural do Rio de Janeiro, Seropédica, 2014.

WHEELER, B. ImPerm: permutation tests for linear models. R package version 1.1-2. 2010. Disponível em: $<$ http://CRAN.R-project.org/package=lmPerm $>$. 